\title{
La patogenesi dell'ipertensione nella malattia policistica
}

\section{A. Zuccalà}

U.O.C. Nefrologia e Dialisi, Imola

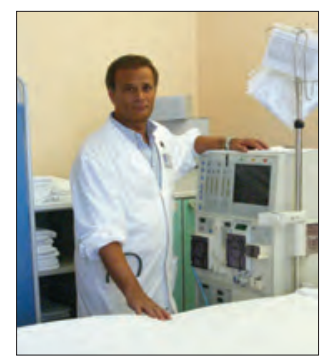

Per dimostrare un rapporto di causa ed effetto tra l'ipertensione e una qualsivoglia malattia renale primitiva, bisogna evitare due importanti fattori confondenti. Il primo è l'elevata prevalenza dell'ipertensione essenziale nella popolazione generale, prevalenza che cresce con l'età fino ad interessare più del 50\% dei soggetti anziani. Il secondo è l'eventuale presenza di insufficienza renale che è un potente ma "aspecifico" fattore di ipertensione. Va considerato, a tal proposito, che 1'85-90\% dei soggetti con insufficienza renale, negli stadi $4^{\circ}$ e $5^{\circ}$ della classificazione internazionale K-DOQI, sono ipertesi.

Tuttavia nel caso della malattia policistica siamo ragionevolmente sicuri che tale entità morbosa sia la vera causa dell'aumento dei valori pressori. Tale affermazione è suffragata da numerosi dati. È ben noto che nei bambini l'ipertensione essenziale è di evenienza eccezionale se non virtualmente assente. Ebbene la pressione arteriosa appare più elevata, in media di 4-6 $\mathrm{mmHg}$, nei bambini con malattia policistica renale autosomica dominate (ADPKD) rispetto ai controlli (1). Il 10-20\% dei bambini con ADPKD mostrano valori pressori superiori $a 5^{\circ}$ percentile (2). Ancora maggiore (34\%) è il tasso di ipertensione, nei bambini con ADPKD, se la PA viene misurata con la registrazione 24 ore (2). Infatti nel 25\% dei bambini con ADPKD è assente il fisiologico calo notturno della PA (2).

Anche per quanto riguarda l'insufficienza renale quale fattore confondente $\mathrm{i}$ dati a favore di un rapporto di casualità tra la $\mathrm{ADPKD}$ e l'aumento dei valori pressori sono piuttosto evidenti. L’ipertensione si manifesta prima della perdita della funzione renale nel $60 \%$ dei soggetti con ADPKD, aumentando al 100\% nellinsufficienza renale stadio $4-5^{\circ} .(3,4)$. Inoltre, l'età media di insorgenza dell'ipertensione si situa tra 30 e i 34 anni di età, molto precocemente rispetto a quanto accade per l'ipertensione essenziale (5). I dati epidemiologici sin qui citati fanno quindi propendere per un rapporto di causa ed effetto tra l'ADPKD e l'ipertensiuone arteriosa, vediamo ora quali sono le caratteristiche dei soggetti con $\mathrm{ADPKD}$ che diventano ipertesi.

\section{Caratteristiche dei soggetti con ADPKD che sviluppano Ipertensione}

I soggetti che sviluppano ipertensione arteriosa rispetto a quelli che rimangono normotesi hanno in media:

- reni di volume maggiore (5)

- un flusso renale plasmatico ridotto (6)

- una maggiore proteinuria (7).

Queste caratteristiche sono particolarmente evidenti nei bambini ipertesi che, oltre a presentare reni di volume maggiore, presentano anche cisti più numerose e con un maggiore incremento di volume nel tempo. Nei bambini inoltre il volume dei reni correla sia con i valori della PA diurna che notturna $(1,2)$.

Le caratteristiche su descritte costituiscono la base di una ipotesi suggestiva e tuttora sostenuta da alcuni autori, secondo la quale le cisti comprimono e distorcono l'architettura vascolare, provocando aree focali di ipossia/ischemia, con attivazione del sistema renina-angiotensina (Fig. 1). A sostegno di tale ipotesi è uno studio angiografico del 1969 che dimostra un rapporto diretto tra il numero e il volume delle cisti e la rarefazione dei vasi renali (8), e il dato che vi è un'aumentata produzione di eritropoietina, espressione di ischemia renale, nei pazienti con ADPKD (9).

Tuttavia i primi studi effettuati per verificare tale ipotesi non evidenziarono un'aumentata attività plasmatica della renina rispetto ai soggetti di controllo $(3,4)$. Chapman et al paragonando a un gruppo di ipertesi essenziali, gli ipertesi con $\mathrm{ADPKD}$, trovavano in questi ultimi 


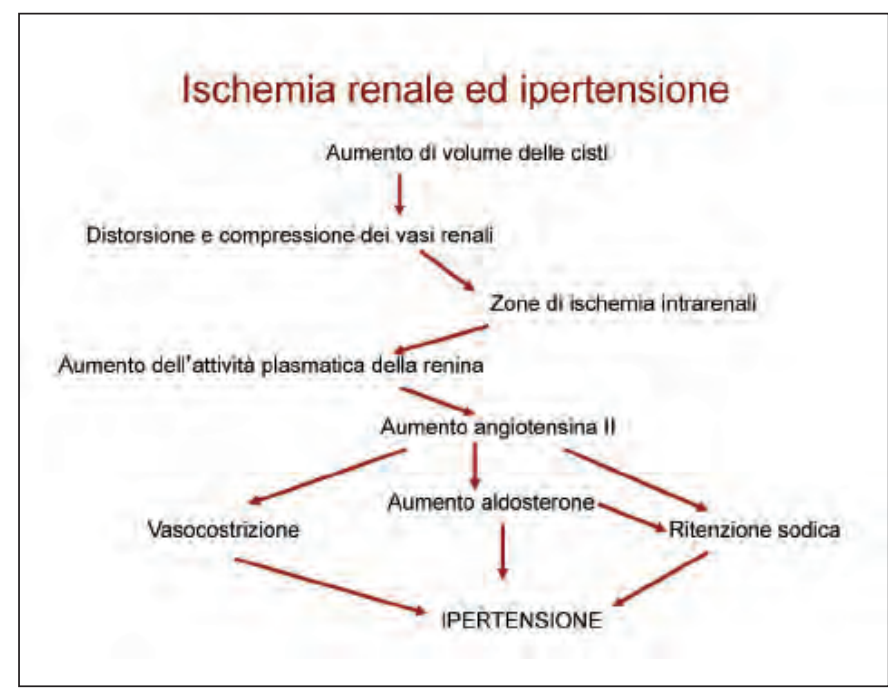

Fig. 1 -

valori più elevati di attività plasmatica della renina, sia in clinostatismo che in ortostatismo (6). Inoltre i valori di APR erano più elevati dopo stimolo con Captopril nei soggetti con ADPKD (6).

I dati di Chapman et al non sono stati peraltro confermati dal gruppo scozzese di Doulton (10) il quale non ha trovato alcuna differenza tra ipertesi essenziali e ipertesi con ADPKD. Una spiegazione della discordanza dei dati tra i due studi potrebbe essere la presenza nella popolazione di ipertesi essenziali, studiata da Chapman, di soggetti afro-americani i quali hanno notoriamente bassi livelli di renina plasmatica.

L'attenzione degli studiosi si è quindi appuntata sul sistema renina-angiotensina (SRA) intrarenale. Graham et al (11) furono i primi a dimostrare un'attivazione del sistema renina angiotensina al di fuori dell'apparato glomerulare, sia nella parete delle arterie che nell'epitelio delle cisti. Tali risultati sono stati confermati da Loghman-Adham et al (12) che identificarono tutti i componenti del SRA e precisamente l'angiotensinogeno, la renina, l'ACE, l'angio II ed il recettore per l'angio II all'interno delle cisti.

Tali autori sostengono che l'epitelio della cisti essendo più indifferenziato rispetto al tubulo renale normale abbia una maggiore espressione del sistema reninaangiotensina. Ricordo che nei tessuti embrionali vi è una vivace attività tissutale del SRA che poi si attenua fino a scomparire nell'adulto. Tale attività anomala del SRA porterebbe a un iper-riassorbimento tubulare del sodio e questo fenomeno provocherebbe l'aumento dei valori pressori (Fig. 2). A favore di tale ipotesi vi sarebbe anche il dato di un aumento del pool sodico scambiabile nei soggetti con ADPKD (13).

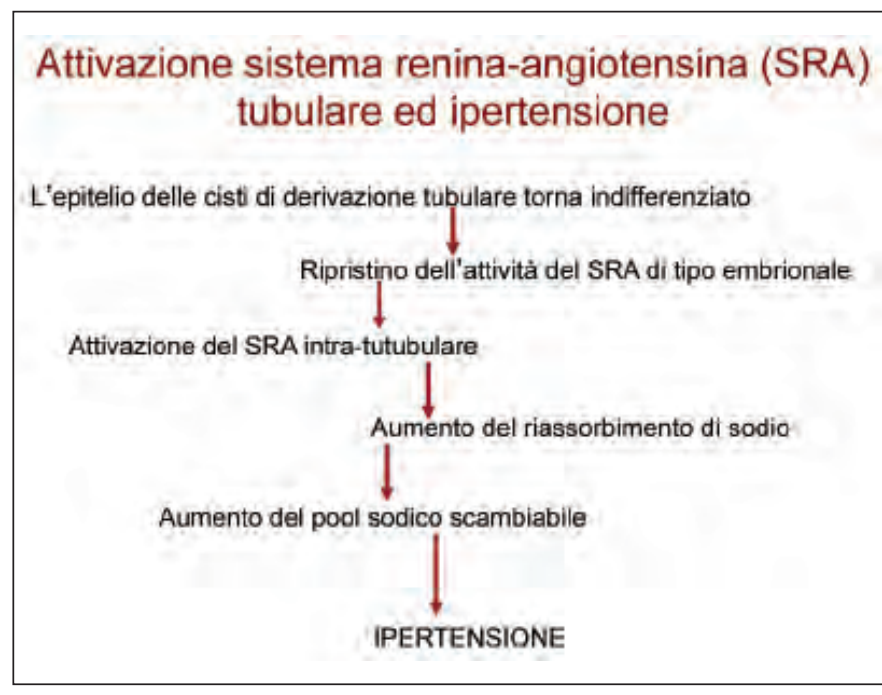

Fig. 2

Vanno infine ricordati alcuni dati sperimentali e clinici a favore del coinvolgimento del sistema simpatico. Gattone VH et al (14) hanno dimostrato che in ratti con ADPKD la denervazione renale, effettuata 4 settimane dopo la nascita, riduceva il volume del rene, il numero e il volume delle cisti, la pressione sistolica e migliorava la funzione renale rispetto ai ratti ADPKD non operati. Nei pazienti con ADPKD, paragonati agli ipertesi essenziali, sono stati dimostrati aumenti delle catecolamine plasmatiche (15), e la registrazione diretta dell'attività nervosa simpatica, a livello del nervo peroneo, ha dimostrato che il numero degli spikes nervosi (tale numero è proporzionale all'attività simpatica periferica) era più alto nei soggetti ipertesi con $\mathrm{ADPKD}$ rispetto ai soggetti normotesi con $\mathrm{ADPKD}$ e ai controlli sani (16).

\section{La terapia con farmaci bloccanti il sistema renina-angiotensina}

Qualche studio retrospettivo aveva dimostrato una superiorità degli ACE-I nel procrastinare l'inizio della dialisi (17). Tuttavia il risultato degli studi randomizzati, che paragonavano l'effetto degli ACE-I verso la terapia standard, sulla progressione dell'IRC nei pazienti con $\mathrm{ADPKD}$ è stato in genere deludente $(18,19)$.

In una meta-analisi su otto studi effettuata da Jafar et al comprendente 142 soggetti con ADPKD si aveva una riduzione del $25 \%$, riguardante un end-point composito di necessità di dialisi e raddoppio della creatinina, a favore degli ACE-I, ma tale differenza non era statisticamente significativa (20).

In uno studio prospettico durato 7 anni in pazienti con 
Alterazione delle policistine ed Ipertensione

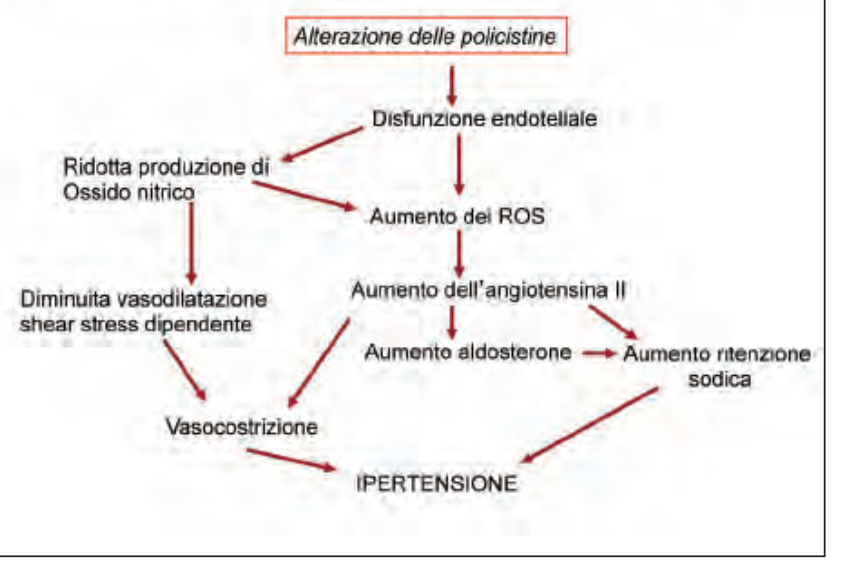

Fig. 3

ADPKD e insufficienza renale, non fu dimostrato alcun beneficio sulla progressione dell'IR con l'uso degli ACE-I versus Ca-antagonisti (21).

Un effetto positivo degli ACE-I sulla proteinuria ma non sulla progressione dell'insufficienza renale, veniva trovato in un piccolo studio randomizzato su $24 \mathrm{ADPKD}$ senza insufficienza renale seguiti per 5 anni (22).

In un altro studio randomizzato di confronto tra un ACEinibitore (Ramipril) e un beta bloccante, gli Autori, dopo 3 anni di follow-up, non furono in grado di dimostrare differenze significative tra $\mathrm{i}$ due farmaci per quanto riguardava la progressione dell'insufficienza renale, l'entità della proteinuria e lo sviluppo di ipertrofia ventricolare sinistra. Tuttavia in tale studio fu dimostrato che un controllo rigoroso della pressione arteriosa portava a una riduzione della proteinuria e dellipertrofia ventricolare sinistra.

Tutti questi studi sono stati sottoposti a critiche di vario tipo, concernenti la numerosità del campione, spesso modesta, la durata del follow-up, spesso limitata nel tempo, e inoltre l'uso di dosi di famaco troppo modeste per bloccare completamente il SRA. In effetti l'aumento dell'attività chimasica nei reni con $\mathrm{ADPKD}$ potrebbe generare meccanismi non ACE dipendenti per la produzione di Angio II (23) e quindi rendere insufficiente l'uso dei soli ACE-I.

Per avere una risposta definitiva e non inficiata da problemi metodologici, ai dubbi che si sono finora accumulati, è stato programmato lo studio HALT .

Lo studio HALT (24) è uno studio multicentrico, randomizzato, doppio cieco, che paragonerà l'effetto di un doppio blocco del SRA con un ACE-I associato ad un bloccante recettoriale dell'angiotensina II (Lisinopril 5 mg, Telmisartan $40 \mathrm{mg}$ ) verso un ACE-I (Lisinopril 5 $\mathrm{mg}$ ) associato a placebo. Tale studio arruolerà 1018 $\mathrm{ADPKD}$, ipertesi. L'arruolamento durerà 3 anni e il follow-up dai 4 agli 8 anni.

Tale studio è tuttora in corso e potrebbe dire una parola definitiva sull'utilità dei farmaci che bloccano il SRA e forse chiarire meglio la fisiopatologia dell'ipertensione nella $\mathrm{ADPKD}$.

\section{Il problema delle policistine vascolari}

Le policistine 1 e 2, proteine prodotte dai geni PKD1 e PKD2, sono importanti per lo sviluppo di un sistema cardiovascolare normale. Infatti ambedue le policistine sono espresse sia nelle cellule endoteliali che muscolari lisce dei vasi (25). Dati sperimentali hanno dimostrato che il topo affetto da una mutazione omozigote del gene PKD1 muore in utero per gravi difetti cardiovascolari (25) e nel topo eterozigote per mutazione del gene PKD2 (PKD2+/-) si hanno anomalie dei vasi intracraniali (26). Le policistine sono importanti anche per la funzionalità vascolare. La vasodilatazione del topo, nel quale il gene PKD1 è stato inattivato, è alterata a causa di un difettoso rilascio dell'ossido nitrico da parte dell'endotelio (26). Anche nell'uomo ci sono prove di alterazioni della vasodilatazione: infatti la vasodilatazione endoteliodipendente appare alterata nei soggetti con ADPKD anche prima dello sviluppo di ipertensione arteriosa (27). Tale alterazione non sarebbe dovuta a una diminuita capacità della fibrocellula muscolare liscia di rispondere all'ossido nitrico ma a una difettosa generazione del gas da parte dell'endotelio. (27). Il rilascio dell'ossido nitrico è modulato da fattori emodinamici. Lo shear stress aumenta il contenuto di calcio dell'endotelio e stimola il rilascio di NO. Ciò serve, da un punto di vista finalistico, a permettere lo scorrimento del sangue verso i tessuti che hanno maggiormente bisogno di ossigeno in quel momento (per esempio, quando corriamo i tessuti che hanno più bisogno di ossigeno sono i muscoli delle gambe). Se tale sistema non è integro si genera ipertensione.

In sostanza viene ipotizzato che il complesso formato dalla policistina 1 e dalla policistina 2 agisca come un meccanotransduttore che "sente" il flusso dei fluidi. Il complesso PC1/PC2 sarebbe quindi un sensore dello scorrimento del sangue eagirebbetramite una complessa catena biochimica per mantenere la circolazione normale (28). Cellule epiteliali che mancano di policistine (o possiedono policistine difettose) non riescono a indurre la risposta tipica quando esposte allo stress circolatorio (28) e quindi non permettono lo scorrimento fisiologico 
del sangue che, incontrando maggiori resistenze del dovuto, genera ipertensione a monte (Fig. 3). Tutto ciò potrebbe spiegare anche perché la riserva funzionale coronarica appare diminuita nei soggetti normotesi con ADPKD (18).

Le policistine, in particolare la policistina 1 , sono particolarmente importanti anche per stabilizzare l'architettura cellulare, processo che appare alterato sia a livello renale che cardiovascolare (29) nei soggetti con ADPKD. Anormalità delle valvole cardiache sono state riportate nel $25 \%$ dei soggetti con ADPKD (3). Aneurismi intracraniali sono presenti nell'8-10\% dei casi (29) con una prevalenza 10 volte più alta della popolazione generale e con una età mediana alla rottura di 44 anni. Inoltre sono stati descritti aneurismi delle coronarie, dell'aorta addominale, dilatazione della radice aortica, dissezioni dell'aorta toracica e delle arterie cervico-cefaliche (7).

Tali alterazioni potrebbero non essere secondarie all'ipertensione precoce e spesso grave, come si è finora pensato, ma sarebbero antecedenti ad essa. Infatti soggetti normotesi con $\mathrm{ADPKD}$ mostrano già un aumento sia della massa del ventricolo sinistro che destro rispetto ai controlli (6) e lo spessore medio- intimale della carotide appare aumentato nei normotesi con $\mathrm{ADPKD}$, in particolare per il contenuto aumentato di tessuto fibroso della parete carotidea (18).

In conclusione, possiamo dire di sapere con ragionevole sicurezza che la ADPKD è in grado di generare ipertensione ma non abbiamo ancora certezze sui meccanismi fini che determinano l'aumento dei valori pressori. Il rischio vascolare potrebbe essere condizionato anche da alterazioni strutturali e/o funzionali dei vasi, che precedono lo sviluppo di ipertensione. La coesistenza di eventuali anomalie vascolari deve quindi indurre medici e pazienti a tenere sotto controllo precocemente e in maniera particolarmente rigorosa i valori pressori.

\author{
Indirizzo degli Autori: \\ Dr. Alessandro Zuccalà \\ Ospedale S. Maria della Scaletta \\ Via Montericco 4 \\ 40026, Imola \\ a.zuccala@ausl.imola.bo.it
}

\section{Bibliografia}

1. Fick-Brosnahan GM, Tran ZV, Johnson AM, Strain JD, Gabow PA. Progression of autosomal-dominant polycystic kidney disease in children. Kidney Int 2001; 59: 1654-62.

2. Rizk D, Jurkovitz C, Veledar E, et al. Quality of life in autosomal dominant polycystic kidney disease patients not yet on dialysis. Clin J Am Soc Nephrol 2009; 4: 560-6.

3. Gabow PA. Autosomal dominant polycystic kidney disease. N Engl J Med 1993; 329: 332-42.

4. Valvo E, Gammaro L, Tessitore N, et al. Hypertension of polycystic kidney disease: mechanisms and hemodynamic alterations. Am J Nephrol 1985; 5: 176-81.

5. Chapman AB, Guay-Woodford LM, Grantham JJ, et al. Renal structure in early autosomal-dominant polycystic kidney disease (ADPKD): The Consortium for Radiologic Imaging Studies of Polycystic Kidney Disease (CRISP) cohort. Kidney Int 2003; 64:1035-45.

6. Chapman AB, Johnson A, Gabow PA, Schrier RW. The renin-angiotensin-aldosterone system and autosomal dominant polycystic kidney disease. N Engl J Med 1990; 323: 1091-6.
7. Chapman AB, Johnson AM, Gabow PA, Schrier RW. Overt proteinuria and microalbuminuria in autosomal dominant polycystic kidney disease. J Am Soc Nephrol 1994; 5: $1349-54$.

8. Ettinger A, Kahn PC, Wise HM Jr. The importance of selective renal angiography in the diagnosis of polycystic disease. J Urol 1969; 102: 156-61.

9. Eckardt KU, Möllmann M, Neumann R, et al. Erythropoietin in polycystic kidneys. JCI 1989; 84: 1160-66.

10. Doulton TW, Saggar-Malik AK, He FJ, et al. The effect of sodium and angiotensin-converting enzyme inhibition on the classic circulating renin-angiotensin system in autosomal-dominant polycystic kidney disease patients. J Hypertens 2006; 24: 939-45.

11. Graham PC, Lindop GB. The anatomy of the renin-secreting cell in adult polycystic kidney disease. Kidney Int 1988; 33: 1084-90.

12. Loghman-Adham M, Soto CE, Inagami T, Cassis L.The intrarenal renin-angiotensin system in autosomal dominant polycystic kidney disease. Am J Physiol Renal Physiol 2004; 287: F775-F788.

13. Harrap SB, Davies DL, Macnicol AM, et al. Renal, car- 
diovascular and hormonal characteristics of young adults with autosomal dominant polycystic kidney disease. Kidney Int 1991; 40: 501-8.

14. Gattone VH 2nd, Siqueira TM Jr, Powell CR, Trambaugh CM, Lingeman JE, Shalhav AL. Contribution of renal innervation to hypertension in rat autosomal dominant polycystic kidney disease. Exp Biol Med 2008; 233: 95257.

15. Cerasola G, Vecchi M, Mulè G, et al. Sympathetic activity and blood pressure pattern in autosomal dominant polycystic kidney disease hypertensives. Am J Nephrol 1998; 18: 391-98.

16. Klein IH, Ligtenberg G, Oey PL, Koomans HA, Blankestijn PJ.Sympathetic activity is increased in polycystic kidney disease and is associated with hypertension. J Am Soc Nephrol 2001; 12: 2427-33.

17. Ecder T, Edelstein CL, Fick-Brosnahan GM, et al. Diuretics versus angiotensin-converting enzyme inhibitors in autosomal dominant polycystic kidney disease. Am J Nephrol 2001; 21: 98-103.

18. Maschio G, Alberti D, Janin G, et al. Effect of the angiotensin-converting-enzyme inhibitor benazepril on the progression of chronic renal insufficiency. The Angiotensin-Converting-Enzyme Inhibition in Progressive Renal Insufficiency Study Group. N Engl J Med 1996; 334: 939-45.

19. Klahr S, Breyer JA, Beck GJ, Dennis VW, Hartman JA, Roth D, Steinman TI<, Wang SR, Yamamoto ME. Dietary protein restriction, blood pressure control and the progression of polycystic kidney disease. Modification of Diet in Renal Disease Study Group. J Am Soc Nephrol 1995; 5(12): 2037-47.

20. Jafar T, Stark PC, Schmid CH, Standgaard S, Kamper A, Maschio G, Becker G, Perrone RD, Levey A. The effect of angiotensin-converting enzyme inhibitors on progression of polycystic kidney disease (PKD) Kidney Int 2005; 6(1): 265-71.
21. Schrier R, McFann K, Johnson A, et al. Cardiac and renal effects of standard versus rigorous blood pressure control in autosomal-dominant polycystic kidney disease: results of a seven-year prospective randomized study. J Am Soc Nephrol 2002; 13: 1733-39.

22. Nutahara K, Higashihara E, Horie S, et al. Calcium channel blocker versus angiotensin II receptor blocker in autosomal dominant polycystic kidney disease. Nephron Clin Pract 2005; 99: c18-23.

23. McPherson EA, Luo Z, Brown RA, et al. Chymase-like angiotensin II-generating activity in end-stage human autosomal dominant polycystic kidney disease. J Am Soc Nephrol 2004; 15: 493-500.

24. Chapman AB, Torres VE, Perrone RD, et al. The HALT Polycystic Kidney Disease Trials - Design and Implementation. Clin J Am Soc Nephrol 2010; 5: 102-09.

25. Boulter C, Mulroy S, Webb S, Fleming S, Brindle K, Sandford R. Cardiovascular, skeletal, and renal defects in mice with a targeted disruption of the Pkd1 gene. Proc Natl Acad Sci U S A. 2001; 98: 12174-79.

26. Hassane S, Claij N, Lantinga-van Leeuwen IS, et al. Pathogenic sequence for dissecting aneurysm formation in a hypomorphic polycystic kidney disease 1 mouse model. Arterioscler Thromb Vasc Biol 2007; 27: 2177-83.

27. Wang D, Iversen J, Wilcox CS, Strandgaard S. Endothelial dysfunction and reduced nitric oxide in resistance arteries in autosomal-dominant polycystic kidney disease. Kidney Int 2003; 64: 1381-88.

28. Nauli SM, Kawanabe Y, Kaminski JJ, Pearce WJ, Ingber DE, Zhou J. Endothelial cilia are fluid shear sensors that regulate calcium signaling and nitric oxide production through polycystin-1. Circulation 2008; 117: 1161-71.

29. Bichet D, Peters D, Patel AJ, Delmas P, Honoré E. Cardiovascular polycystins: insights from autosomal dominant polycystic kidney disease and transgenic animal models. Trends Cardiovasc Med 2006; 16: 292-8. 